\title{
Laringectomía total ampliada en carcinoma laríngeo avanzado T4a*
}

\author{
Drs. RICARDO YÁÑEZ M. ${ }^{1}$, FRANCISCO JAVIER LOYOLA B. ${ }^{1,2}$, \\ Al. CAMILO URZÚA E. ${ }^{3}$, Dr. JORGE CORNEJO F. ${ }^{1}$ \\ 1 Cirugía de Cabeza y Cuello. Servicio de Cirugía. Hospital Dr. Sótero del Río. \\ 2 Pontificia Universidad Católica de Chile. \\ 3 Alumno de Medicina. Universidad de Santiago. \\ Santiago, Chile.
}

\begin{abstract}
Total laryngectomy in advanced laryngeal neoplasma T4a

Background: In Chile a significant number of patients is presented with advanced laryngeal cancer at diagnosis with poor prognosis and less than $50 \%$ survival at 5 years. Nowadays choosing the organ sparing treatment, but in selected cases total laryngectomy is a valid treatment option. Aim: To present the results obtained with the extended total laryngectomy in patients with advanced laryngeal carcinoma. Patients and Methods: Retrospective analysis of all patients with advanced laryngeal carcinoma T4a underwent total extended laryngectomy at our center between 2007-2010. Results: 18 patients, a woman, a median age 64 years (range, 50-82), they were studied and etapified with Rhino-Laryngo-Fibrescope Olympus@ and CT. Seven patients underwent emergency tracheostomy. All patients underwent total extended laryngectomy with primary pharyngeal closure. The median tumor size was $4.3 \mathrm{~cm}$. In 17 patients R0 was achieved. Postoperative complication was presented in one patient with a pharyngocutaneous fistula. Thirteen patients were sent to radiotherapy. After a follow-up of 17 months (range, 2-55): one, two and three years of survival was $88.8 \%$, $50 \%$ and $50 \%$ respectively. Conclusion: In the serie, extended total laryngectomy is a good therapeutic alternative in patients with advanced laryngeal carcinoma, with low surgical morbidity, short hospital stay and favorable survival.
\end{abstract}

Key words: Laryngeal neoplasms, laryngectomy, organ sparing treatments, neoplasm invasiveness.

\section{Resumen}

Introducción: En Chile se presenta un importante número de pacientes con cáncer laríngeo avanzado al momento del diagnóstico, con mal pronóstico y una supervivencia menor al $50 \%$ a 5 años. Actualmente, se opta por el tratamiento conservador de órganos, en casos seleccionados la laringectomía total es una alternativa. Objetivo: Presentar los resultados de la laringectomía total ampliada en carcinoma laríngeo avanzado. Pacientes y Método: Análisis retrospectivo de los pacientes con carcinoma laríngeo avanzado T4a sometidos a laringectomía total ampliada en nuestro centro entre 2007-2010. Resultados: La serie estuvo constituida

*Recibido el 16 de abril de 2012 y aceptado para publicación el 7 de junio de 2012.

Los autores no refieren conflictos de interés.

Correspondencia: Dr. Ricardo Yáñez M.

Av. Concha y Toro 3459, Puente Alto, Santiago, Chile. Fax: +56-2-3536601

yanezmartinez@gmail.com 
por 18 pacientes, una mujer, mediana de 64 años (rango, 50-82), estudio y etapificación con nasofibroscopia y TC. En siete pacientes se realizó traqueostomía de urgencia. En todos los pacientes se realizó laringectomía total ampliada. El tamaño tumoral fue 4,3 cm como mediana. En 17 pacientes se logró R0. Como complicación un paciente presentó una fístula faringocutánea. Fueron enviados a radioterapia 13 pacientes. Tras un seguimiento de 17 meses (rango, 2-55): la supervivencia a uno, a dos y a tres años fue de 88,8\%, 50\% y 50\% respectivamente. Conclusión: En esta serie, la laringectomía total ampliada es una buena alternativa para los pacientes con carcinoma laríngeo avanzado T4a por su baja morbilidad quirúrgica, corta estadía hospitalaria y supervivencia favorable.

Palabras clave: Cáncer laríngeo, laringectomía, tratamiento conservador de órganos.

\section{Introducción}

En Chile, el cáncer de laringe es la primera causa de muerte dentro de los tumores malignos de cabeza y cuello, no existen datos sobre la incidencia, pero la tasa de mortalidad bruta en nuestro país es de 0,9/100.000 habitantes, una mortalidad específica que va de 0,04/100.000 en menores de 50 años a $10,97 / 100.000$ en mayores de 80 años; con una razón hombre:mujer de 6:1 ${ }^{1}$. Mundialmente la incidencia ha disminuido en hombres, pero se mantiene en ascenso en mujeres ${ }^{2}$.

El tabaco y el alcohol son los factores de riesgo más frecuentemente asociados con el desarrollo de cáncer laríngeo, lo que unido a una consulta tardía, provoca que más de la mitad de los pacientes sea diagnosticado en una etapa avanzada ${ }^{3}$.

Según la ubicación en la laringe se puede determinar tres ubicaciones; correspondiendo el cáncer de supraglotis al 35\%, el cáncer de glotis al 60\% y el cáncer subglótico al 5\%, lo que conlleva diferencias en su presentación, estudio y tratamiento ${ }^{4}$.

El objetivo del tratamiento es la erradicación de la enfermedad con los criterios de terapia conservadora de órganos cuando es posible ${ }^{5}$. En tumores glóticos T1 y T2 la cirugía y la radioterapia tienen excelentes resultados, con una sobrevida a cinco años de 80 a 90\%, mientras que para las lesiones T3 y T4 la sobrevida es de 50 a $60 \%{ }^{6,7}$. Según la clasificación del American Joint Committee on Cancer, los tumores de glotis en estadio T4a son aquellos que invaden a través de la superficie externa del cartílago tiroides y/o invaden distintos tejidos alrededor de la laringe (como por ejemplo la traquea, partes blandas del cuello incluyendo musculatura extrínseca profunda de la lengua, tiroides o esófago $)^{8}$; en este grupo de pacientes con enfermedad más avanzada está indicada la laringectomía total asociada a una disección cervical bilateral y está indicada la tiroidectomía total o hemitiroidectomía en aquellos casos donde existe compromiso transglótico, tumores subglóticos y tumores con extensión subglótica mayor a $10 \mathrm{~mm}^{9}$. En casos con extensión importante a hipofaringe o a esófago cervical puede realizarse una laringofaringectomía. La radioterapia posto- peratoria es parte del tratamiento en pacientes con cáncer laríngeo avanzado, es decir, pacientes con extensión supra o subglótica o en aquellos pacientes que presentan compromiso extralaríngeo; también está indicada resecciones con márgenes positivos, linfonodos positivos múltiples y pacientes que requirieron traqueostomía prelaringectomía por el mayor riesgo de recurrencia en el estoma ${ }^{10,11}$.

Este grupo de pacientes con patología avanzada y sometidos a una modalidad terapéutica combinada, es decir, laringectomía total y quimiorradioterapia postoperatoria logran una sobrevida a cinco años no mayor que $50 \%{ }^{11}$.

El objetivo de este estudio es presentar los resultados obtenidos con la laringectomía total ampliada en pacientes con carcinoma laríngeo avanzado T4a.

\section{Pacientes y Método}

\section{Pacientes}

Se realizó un análisis retrospectivo de todos los pacientes con carcinoma laríngeo avanzado, es decir, en estadio T4a sometidos a laringectomía total ampliada en el Hospital Dr. Sótero del Río entre junio de 2007 y diciembre de 2010.

Se realizó revisión de fichas clínicas, se determinó los siguientes datos de los pacientes: género, edad, hábito tabáquico y alcohólico, motivo de consulta, necesidad de traqueostomía de urgencia, diagnóstico preoperatorio, cirugía realizada, sobrevida (en meses). Se registró estadía hospitalaria, complicaciones (tempranas y tardías), inicio de alimentación enteral/oral y tratamiento adyuvante.

Las complicaciones postoperatorias tempranas fueron definidas como aquellas que se presentaron dentro de los primeros 90 días desde la cirugía ${ }^{12}$. Además se determinó recidiva y necesidad de reoperación.

Desde el punto de vista anatomopatológico se estableció el tamaño tumoral, tipo histológico, grado de diferenciación, estado de los márgenes quirúrgicos, estado de los ganglios, permeación vascular e invasión de la glándula tiroides.

Con una búsqueda en el Servicio de Registro Civil se determinó el dato de mortalidad. 


\section{Resultados}

La serie está compuesta por 18 pacientes, una mujer. La mediana de edad fue de 64 años (rango, 50-82 años). En 16 pacientes (88,8\%) se obtuvo como antecedente un hábito tabáquico y en 11 pacientes (61\%) un consumo excesivo de alcohol (Tabla 1).

En 13 pacientes (72,2\%) el motivo de consulta fue la disfonía, seguido por la disnea en siete pacientes $(38,8 \%)$. Todos los pacientes fueron estudiados y etapificados preoperatoriamente con Nasofibroscopio Olympus ${ }^{\circledR}$ y tomografía axial computada (TC) de cabeza y cuello.

En siete pacientes $(38,8 \%)$ se debió realizar una traqueostomía de urgencia por estenosis crítica de la vía aérea.

En todos los pacientes se realizó una laringectomía total ampliada, es decir, faringolaringectomía total con disección cervical bilateral y tiroidectomía del lado del tumor con cierre faríngeo primario. Se encuentra disponible un vídeo resumido de la técnica utilizada en nuestro centro en www.medicalvideos.us/play.php?vid $=4071$

Según las características anatomopatológicas del tumor laríngeo, el tamaño tumoral promedio fue de $4 \mathrm{~cm}$ (rango, 2-6 cm) y en 17 pacientes $(94,4 \%$ ) los bordes quirúrgicos fueron negativos. En todos los pacientes la ubicación del tumor fue glótica.

De acuerdo a la histología de la neoplasia, todos los pacientes presentaron un carcinoma escamoso, de los cuales ocho pacientes $(44,4 \%)$ fueron informados como bien diferenciado y 10 pacientes (55,5\%) como moderadamente diferenciado; cuatro pacientes $(22,2 \%)$ presentaron permeacion vascular. Asimismo, el estadio ganglionar fue positivo en ocho pacientes $(44,4 \%)$ y sólo un paciente $(5,5 \%)$ presentó invasión tiroidea.

No hubo mortalidad postoperatoria. La complicación presentada fue en un paciente con una fístula faringocutánea con manejo médico exitoso.

La realimentación enteral se realizó a las 48 h por sonda nasogástrica y a los 12 días (rango, 10-15 días) por vía oral. La estadía hospitalaria fue de 14 días (rango, 11-45 días).

Todos los pacientes fueron presentados en el Comité de Oncología y tres pacientes fueron enviados a quimioradioterapia, diez sólo a radioterapia y a los cinco pacientes restantes se decidió no realizar tratamiento adyuvante por el compromiso del estado general que presentaban.

Posteriormente, tras un seguimiento de 17 meses (rango, 2-55 meses) la supervivencia global al año, a los dos años y a los tres años fue de $88,8 \%, 50 \%$ y $50 \%$ respectivamente (Figura 1 ).

En los pacientes que no fueron sometidos a
Tabla 1. Características de los pacientes con carcinoma laríngeo avanzado sometidos a laringectomía total ampliada

\begin{tabular}{|c|c|c|}
\hline Características de los pacientes & $n=18$ & \\
\hline Edad (mediana y rango) & $\begin{array}{l}64 \text { años } \\
(50-82)\end{array}$ & \\
\hline Género Masculino : Femenino & $17: 1$ & \\
\hline Tabaquismo & 16 pacientes & $88,8 \%$ \\
\hline Alcoholismo & 11 pacientes & $61,1 \%$ \\
\hline $\begin{array}{l}\text { Motivo de consulta } \\
\text { Disfonía } \\
\text { Disnea }\end{array}$ & $\begin{array}{r}13 \text { pacientes } \\
7 \text { pacientes }\end{array}$ & $\begin{array}{l}72,2 \% \\
38,8 \%\end{array}$ \\
\hline $\begin{array}{l}\text { Estenosis crítica de la vía aérea } \\
\text { (Traqueostomía de urgencia) }\end{array}$ & 7 pacientes & $38,8 \%$ \\
\hline
\end{tabular}

adyuvancia con radioterapia la supervivencia al año y a los dos años fue de $60 \%$ y $0 \%$ respectivamente. En los pacientes sometidos a radioterapia la supervivencia al año, a los dos años y a los tres años fue de $100 \%, 50 \%$ y $50 \%$ respectivamente (Figura 2 ).

Según el grado de diferenciación tumoral se obtuvo que la supervivencia al año, a los dos y a los tres años en pacientes con tumores bien diferenciados fue de $100 \%, 75 \%$ y $75 \%$ respectivamente; mientras que en los pacientes con tumores moderadamente diferenciados fue al año y a los dos años de $75 \%$ y 0\% (Figura 3).

En tres pacientes (20\%) hubo recurrencia locorregional tratada con radioterapia.

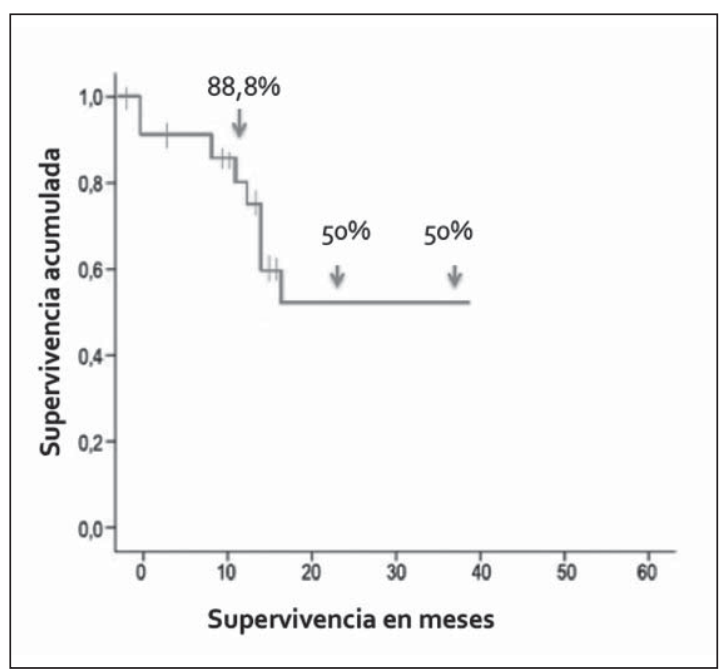

Figura 1. Supervivencia acumulada en pacientes con carcinoma laríngeo avanzado sometidos a laringectomía total ampliada. 

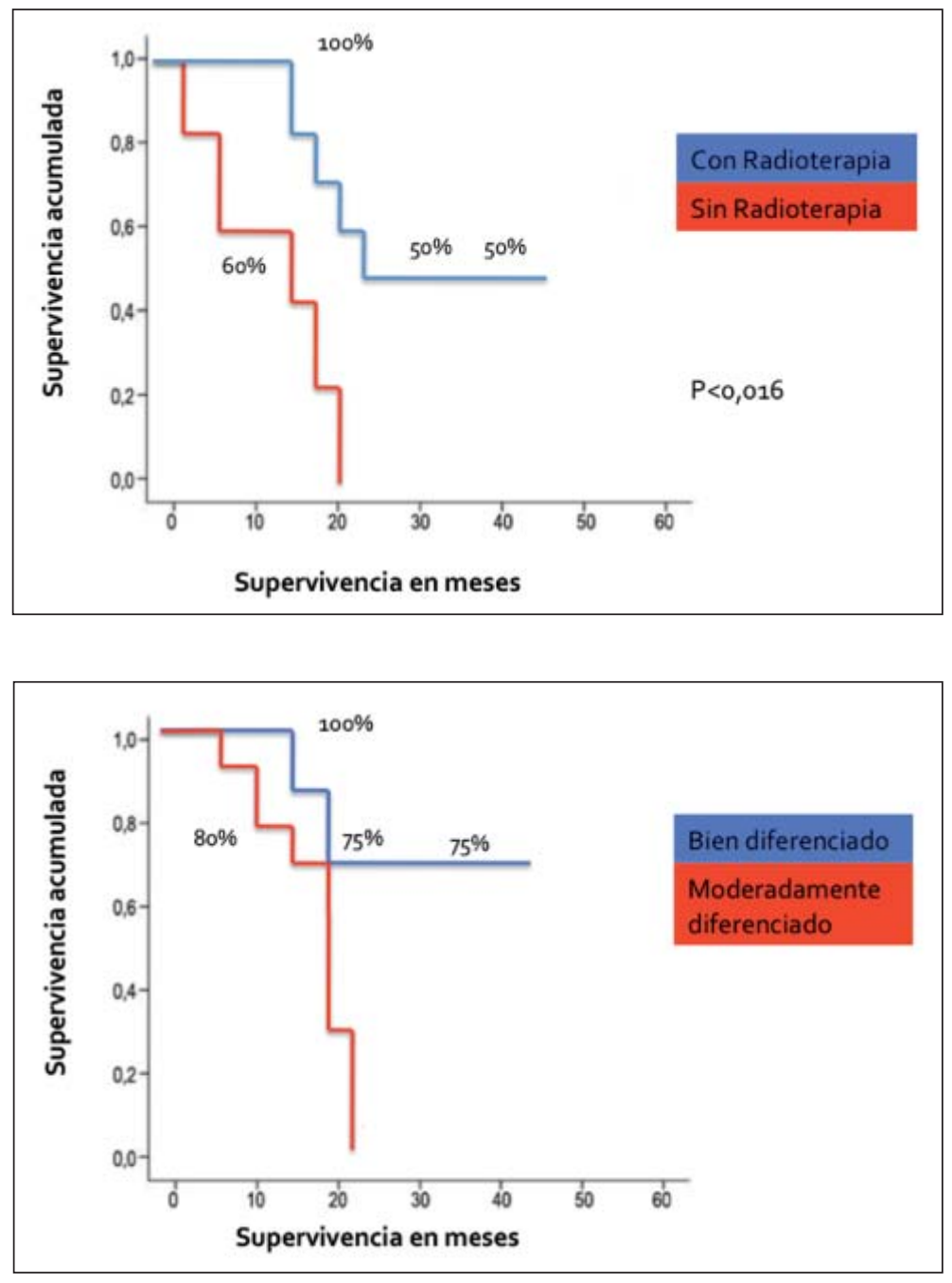

Figura 2. Supervivencia en pacientes con carcinoma laríngeo avanzado sometidos a laringectomía total ampliada con y sin radioterapia adyuvante.

Figura 3. Supervivencia acumulada en pacientes con carcinoma laríngeo avanzado sometidos a laringectomía total ampliada según diferenciación tumoral.

\section{Discusión}

Los tumores glóticos en estadío temprano pueden ser tratados efectivamente con cirugía o radioterapia. Las opciones quirúrgicas para tumores T1 y T2 incluyen resección de cuerda vocal, microcirugía láser transoral, hemilaringectomía y laringectomía subtotal. Se obtienen beneficios con el tratamiento quirúrgico tales como la extirpación tumoral (R0) y reserva de otros tratamientos, como la radioterapia sólo en caso de recurrencias ${ }^{4,13}$. La laringectomía total en general se reserva para el cáncer laríngeo más avanzado (T3 y T4) o para pacientes con mala respuesta a tratamientos previos y para aquellos sometidos a una cirugía conservadora de órganos con pobres resultados funcionales en cuanto a fonación y/o deglución ${ }^{14}$. En general los pacientes con enfermedad avanzada son sometidos a radioterapia postoperatoria asociada o no a quimioterapia ${ }^{14,15}$.

Las características epidemiológicas del cáncer laríngeo avanzado observadas en nuestra serie son presencia mayoritaria en pacientes de sexo masculino asociada a edad sobre los 50 años, además de un claro hábito tabáquico y alcohólico.

Acorde a las características tumorales la sobrevida acumulada es similar a la comunicada en la literatura internacional, donde se señala que los factores más importantes que afectan la sobrevida son la edad del paciente, la etapificación y diferenciación tumoral y los márgenes quirúrgicos ${ }^{7,16}$.

No fue realizado análisis estadístico al evaluar la sobrevida según grado de diferenciación tumoral ni por haber recibido o no radioterapia adyuvante ya que el número de pacientes no permitió separarlos en grupos comparables estadísticamente.

La invasión de la glándula tiroides en pacientes 
sometidos a laringectomía total es poco frecuente y la tiroidectomía total no estaría indicada tal como lo sugieren publicaciones al respecto donde la conservación de la glándula tiroides se asocia a menor morbilidad operatoria sin comprometer el control local de la enfermedad ${ }^{17}$.

El tratamiento del cáncer de laringe avanzado a través de la laringectomía total ampliada es, en nuestro centro, una buena alternativa terapéutica por su baja morbilidad, corta estadía hospitalaria y supervivencia favorable.

\section{Referencias}

1. Bogado ME, Araya FS, Alonso FT. Análisis de la mortalidad por cáncer laríngeo en Chile (1990-2004) Acta Otorrinolaringol Esp. 2007;58:336-40.

2. IARC. Cancer incidence in five continents. Volume VIII. IARC scientific publications. 2002;155:1-781.

3. Riedel F, Goessler UR, Hormann K. Alcohol-related diseases of the mouth and throat. Dig Dis. (Basel) 2005;23:195-203.

4. Newkirk KA, Holsinger FC. Cancers of the Head and Neck. En: Barry W. Feig, C. Denise Ching ed. M.D. Anderson Surgical Oncology Handbook. Fifth edition, 2011. Texas: Editorial: Lippincott Williams \& Wilkins, 2011;196-219.

5. Pfister DG, Laurie SA, Weinstein GS, Mendenhall WM, Adelstein DJ, Kian Ang K, et al. American Society of Clinical Oncology Clinical Practice Guideline for the Use of Larynx-Preservation Strategies in the Treatment of Laryngeal Cancer. Journal of Clinical Oncology 2006:24;3693-704.

6. Celedón C, Royer M, Ortúzar L. Laringectomía parcial en cáncer laríngeo precoz: 25 años de experiencia. Rev. Otorrinolaringol Cir Cabeza Cuello 2008;68:117-23.

7. Papadas TA, Alexopoulos EC, Mallis A, Jelastopulu E, Mastronikolis NS, Goumas P. Survival after laryngectomy: a review of 133 patients with laryngeal carcino- ma. Eur Arch Otorhinolaryngol. 2010;267:1095-101.

8. Edge SB, Byrd DR, Compton CC. ed. AJCC Cancer Staging Manual. 7th ed. New York, NY: Editorial: Springer, 2010;57-67.

9. Mendelson AA, Al-Khatib TA, Julien M, Payne RJ, Black MJ, Hier MP. Thyroid gland management in total laryngectomy: meta-analysis and surgical recommendations. Otolaryngol Head Neck Surg. 2009;140:298-305.

10. Spector GJ, Sessions DG, Lenox J, Newland D, Simpson J, Haughey BH. Management of stage IV glottic carcinoma: therapeutic outcomes. Laryngoscope 2004; 114:1438-46.

11. Ganly I, Patel SG, Matsuo J, Singh B, Kraus DH, Boyle $\mathrm{J}$, et al. Analysis of postoperative complications of open partial laryngectomy. Head Neck 2009;31:338-45.

12. Dindo D, Demartines N, Clavien PA. Classification of surgical complications: a new proposal with evaluation in a cohort of 6336 patients and results of a survey. Ann Surg. 2004;240:205-13.

13. Klozar J, Cada Z, Koslabova E. Complications of total laryngectomy in the era of chemoradiation. Eur Arch Otorhinolaryngol. 2011;269:289-93.

14. Van der Putten L, De Bree R, Kuik DJ, Rietveld DH, Buter J, Eerenstein SE, et al. Salvage laryngectomy: oncological and functional outcome. Oral Oncol. 2011;47:296-301.

15. Bernier J, Domenge C, Ozsahin M, Matuszewska K, Lefèvre JL, Greiner R, et al. Postoperative Irradiation with or without Concomitant Chemoterapy for Locally Advanced Head and Neck Cancer. N Engl J Med. 2004;350:1945-52.

16. Liu TR, Yang AK, Chen FJ, Zeng MS, Song M, Guo $\mathrm{ZM}$, et al. Survival and prognostic analysis of 221 patients with advanced laryngeal squamous cell carcinoma treated by surgery. Ai Zheng 2009;28:297-302.

17. Elliott MS, Odell EW, Tysome JR, Connor SE, Siddiqui A, Jeannon JP, et al. Role of thyroidectomy in advanced laryngeal and pharyngolaryngeal carcinoma. Otolaryngol Head Neck Surg. 2010;142:851-5. 